\title{
Las políticas de desarrollo profesional del profesor principiante en el Programa de Acompañamiento de Docentes Noveles en su Primera Inserción Laboral de Argentina*
}

ANDREA ALLIAUD

Universidad de Buenos Aires,

Buenos Aires, Argentina

\section{COMENZAR A SER DOCENTE HOY}

El período de inserción (de iniciación) laboral resulta complejo en cualquier ámbito de desempeño. Lo que uno sabe, lo que uno aprendió en las instancias formativas que atravesó, nunca parece ser suficiente en los comienzos. Tratándose de ocupaciones en las que además se involucran las "almas" de las personas (la educación es una de ellas) la complejidad aumenta.

Efectivamente, la enseñanza puede caracterizarse como una actividad (no la única) que se ejerce con otros y sobre otros. Durante muchos años este trabajo sobre los otros se anclaba en el denominado "programa institucional" ${ }^{1}$ de la modernidad

* Texto apresentado em Sessão Especial na 35a Reunião Anual da Associação Nacional de Pós-Graduação e Pesquisa em Educação (ANPEd), realizada em Natal/RN, de 2 a 5 de outubro de 2011.

1 "Puede definirse el programa institucional como el proceso social que transforma valores y principios en acción y en subjetividad por el sesgo de un trabajo profesional específico y organizado" (Dubet, 2006, p. 32). 
(Dubet, 2006). Como modo de socialización, este programa consideraba que el trabajo sobre los otros consistía en una mediación entre valores universales e individuos particulares. Los valores (República/razón y civilización) sostenían una actividad basada en el supuesto fundamental que correlacionaba la socialización de los individuos, con el logro de su libertad y autonomía. Acordémonos del proceso de creación y consolidación de los sistemas educativos nacionales, junto con el surgimiento de instancias especializadas para la transmisión del oficio de enseñar.

La fuerte institucionalización que por muchos años sostuvo a este proceso de intervención sobre las personas, hoy está en declive. El programa institucional de la modernidad se encuentra actualmente en decadencia, "resquebrajado", lo cual no implica que no funcione, sino más bien que se halla debilitado y, por lo tanto, lo que antes estaba asegurado institucionalmente ahora tiene que ser garantizado por los sujetos. De este modo, enseñar hoy (intervenir sobre las personas hoy) es menos el cumplimiento de un rol que la construcción de una experiencia (idem, ibidem). Ello significa que lo que antes estaba garantizado por la institución escolar ahora tienen que lograrlo los sujetos que enseñan cotidianamente. Hasta no hace tanto, por el sólo hecho de ocupar "el lugar de" (maestro o profesor) se era reconocido, respetado, escuchado, admirado... y podríamos continuar. Actualmente, para poder enseñar, maestros y profesores tienen que poder construir (con los otros) condiciones que ya no están dadas automáticamente ni legitimadas trascendentalmente, más allá de ellos.

Con lo dicho hasta aquí podemos sostener que:

1. Los comienzos laborales siempre son difíciles.

2. La dificultad aumenta en los casos en que hay personas involucradas.

3. La debilidad institucional, propia de estos tiempos, y el mayor nivel de exposición personal que trae aparejado añade nuevos problemas.

Las formas de acceso a la docencia, recurrentes entre los distintos países, es un cuarto elemento a considerar. A diferencia de otras profesiones, quienes comienzan a enseñar lo hacen, por lo general, en las escuelas más difíciles y en los grados más complejos. La entrada a instituciones de zonas desfavorecidas y a los primeros grados, resultan modalidades de empleo corrientes para los jóvenes docentes. Así, la lógica de aprendizaje (aquella que probablemente maestros y profesores aprendieron durante su formación inicial) se invierte. En lugar de empezar por lo más simple para llegar progresivamente a lo más complejo, maestros y profesores suelen transitar sus primeros años de desempeño en instituciones "complejas" y una vez que están en condiciones (por años trabajados, capacitaciones realizadas, carrera professional, etc.) de poder elegir, recién ahí, pueden optar por escuelas mejor ubicadas, mejor dotadas, menos problemáticas y dentro de ellas también les será más fácil escoger su año/grado de preferencia. La escasa estabilidad laboral dada por la rotación de escuelas junto con la precariedad de las formas de contratación, resultan ser asimismo moneda corriente entre los nuevos docentes. El trabajo en 
soledad, aislado, característico de la docencia, deja a los nuevos en condiciones de desventaja para insertarse, aprender y formarse en sus lugares de trabajo.

De este modo, si enseñar no es fácil (nunca lo fue) menos aún lo es en los comienzos y bajo las condiciones laborales e institucionales en las que actualmente maestros y profesores empiezan a trabajar. Como contrapartida, las renuncias y las deserciones se acrecientan en esta etapa laboral.

A partir de este breve panorama se entiende por qué desde hace no tantos años es tema/"problema" la inserción laboral/profesional de los noveles docentes. Cada vez se insiste más en la importancia de desarrollar programas de formación y desarrollo profesional específicos para quienes se inician en la enseñanza. Y cada vez, de hecho, se desarrollan experiencias y se formulan políticas educativas ${ }^{2}$ que contemplan las problemáticas específicas de este grupo de docentes en particular.

En lo que sigue de este trabajo, nos dedicaremos en primer lugar a presentar ciertas reflexiones surgidas del Programa de Acompañamiento de Docentes Noveles en su Primera Inserción Laboral de Argentina, impulsado por el Ministerio de Educación Nacional desde el año 2005 hasta el presente para, seguidamente, dar la palabra a los docentes noveles que han participado de la experiencia. ${ }^{3} \mathrm{Hacia}$ el final, retomaremos algunas cuestiones fundamentales referidas al oficio docente, su formación y desarrollo profesional.

\section{ACOMPAÑANDO A LOS NUEVOS}

En Argentina son las instituciones superiores de formación docente (IFD) las que asumen la función de desarrollo profesional centrada en los comienzos de la profesión. ${ }^{4}$ Específicamente, son los formadores de maestros y profesores los

2 En Iberoamérica, por mencionar sólo algunos de ellos, podemos referir a los programas desarrollados en: Uruguay (El acompañamiento a los noveles maestros, Dirección de Formación y Perfeccionamiento Docente de la Administración Nacional de Educación Publica); Chile (Proyecto de Inserción de Docentes Noveles en la Educación Municipalizada de Valparaíso- y diversos Proyectos de Mentoría llevados a cabo en distintas universidades); Ecuador (Programa de Mejoramiento de la Calidad Educativa mediante procesos de Mentoría o Acompañamiento pedagógico en el aula, Sistema Integral de Desarrollo Profesional Educativo [SíProfe] de la Subsecretaría de Desarrollo Profesional Educativo, Ministerio de Educación); España (Universidad de Sevilla). Los programas llevados a cabo en el continente europeo, de carácter local, regional o nacional pueden verse en: Pat Ashby et al. Beginner teacher's experiences of initial teacher preparation, induction and early profesional development: a review of literatur. London: DCSF, 2008.

3 La información que se presenta en los puntos que siguen, se detalla en: Acompañar los primeros pasos en la docencia (Alen; Allegroni, 2009). Serie de seis tomos en los que se registra la experiencia de Argentina gracias al trabajo colaborativo entre el área de Formación Docente de la Organización de Estados Iberoamericanos (OEI) de Argentina y el Instituto Nacional de Formación Docente (INFD) del Ministerio de Educación de la Nación.

4 La experiencia de Argentina se diferencia así de la de los países en los que esta función es ejercida por los maestros y profesores de las escuelas en que los nuevos se insertan 
encargados de "acompañar" a los docentes noveles en su primer año de desempeño profesional. Si bien son las mismas instituciones formadoras las que realizan esta tarea, la lógica del acompañamiento representa una ruptura respecto de la formación inicial. Tal como está planteado, el acompañamiento no implica alargar la formación inicial un año más. Los iniciados a la docencia son considerados "trabajadores" que están empezando (no estudiantes) y, por lo tanto, el proceso formativo que con ellos se emprende contempla las problemáticas particulares que les plantea su experiencia laboral.Los aportes de la formación inicial se articulan en función de estas realidades, a la vez que se van generando y poniendo en marcha dispositivos formativos especialmente diseñados que se apoyan en sus preocupaciones y necesidades.

La co-observación de clases, hecha entre pares (docentes noveles) pero guiada por los formadores; los seminarios, centrados en cuestiones disciplinares pero también en problemáticas comunes propias del trabajo pedagógico; los talleres de práctica, en los que se trabaja en pequeños grupos situaciones que presentan dificultades; los ateneos didácticos, en los que se abordan los problemas de enseñanza que los noveles detectan con los alumnos que efectivamente trabajan, son algunos de los dispositivos que se utilizan en el acompañamiento. La escritura de textos de la práctica a través de distintos géneros que se propone a los docentes noveles y a sus formadores, tiene la intención de posibilitar procesos de pensamiento y reflexión tanto individuales como colectivos. Además del trabajo con los noveles docentes, el programa sostiene instancias de articulación con las escuelas en que ellos enseñan, lo cual moviliza y potencia los recursos allí existentes.

La intervención, así plantada, no sólo representa un aporte para los sujetos y las instituciones de referencia. El hecho de que sean los mismos institutos formadores los que realizan el acompañamiento, brinda la posibilidad de enriquecer los procesos formativos que en ellos se emprenden. El contacto permanente con la realidad de las escuelas y las problemáticas de los docentes es un valioso insumo para la formación inicial, cuya principal carencia o debilidad ha sido históricamente la falta de relación o conexión con las problemáticas del trabajo docente. En tiempos en que, como dijimos, la enseñanza se vuelve más compleja, esta "debilidad" merece ser considerada. Concretamente, los casos, las situaciones, las problemáticas que los formadores de docentes detectan en el acompañamiento, representan un material valioso que puede capitalizarse en las distintas instancias de la preparación profesional de los futuros docentes.

\section{UNA FORMA PARTICULAR DE HACER POLÍTICA}

El Ministerio de Educación Nacional viene desarrollando (desde el año 2005) actividades sostenidas destinadas a fortalecer el trabajo de los formadores de docentes involucrados en el Programa de Acompañamiento de las distintas

(mentorazgo, tutorías, etc.) y también de la que llevan a cabo las universidades a través de programas de apoyo o cursos de educación continua. 
jurisdicciones del país. ${ }^{5}$ En la actualidad son más de cuatrocientos los formadores comprometidos, pertenecientes a quince provincias argentinas.

En sus comienzos, el programa se inició como una experiencia piloto en una región particular del país, reconociendo la necesidad de "avanzar en el tránsito entre la formación inicial y el desempeño de los maestros y profesores en sus primeras inserciones laborales" (Alen; Allegroni, 2009, p. 24). Progresivamente, el eje de intervención se fue desplazando hacia la escuela, entendida como lugar de trabajo y de formación cotidiana para los docentes. Considerando el tipo de establecimientos en que los noveles maestros comienzan a trabajar ("complejos", como vimos, la mayoría en situación de vulnerabilidad), se ha intentado también mejorar la situación educativa de los niños y jóvenes que a ellos concurren. Desde su lanzamiento, el programa se basó en la experiencia francesa, desarrollada por ese entonces por Patrice Rayou y sus colaboradores en el Institut Universitaire de Formation des Maîtres (IUFM) de Créteil, con quienes se han formalizado y desarrollado actividades de cooperación (idem).

En el año 2007, con la creación del INFD, la línea de acompañamiento a docentes noveles se constituyó como política nacional dentro del Área de Desarrollo Profesional Docente. Nuevos institutos y nuevas provincias se fueron sumando "voluntariamente" a partir de entonces, lo cual implicó nuevas necesidades; entre ellas, la creación de marcos de intervención no sólo pedagógicos, sino también normativos y organizacionales.

Es de destacar, en primer lugar, cómo se generó el proceso. Partiendo de una experiencia acotada y puntual, fue posible dar forma y formular una política nacional que aún se sigue fortaleciendo y conformando en su devenir. Fue la propia experiencia transitada por los actores (y registrada) lo que sentó las bases para la formulación una política pública que contempla el desarrollo profesional para docentes que se inician y, a la vez, fortalece al sistema educativo. La importancia otorgada al incremento de las relaciones entre el sistema formador de docentes y las escuelas donde éstos se insertan a trabajar (prevista entre las funciones del INFD por la nueva Ley de Educación Nacional de 2007 en su artículo 77), potenció la línea de acompañamiento a los docentes principiantes pues los beneficiarios privilegiados de este trabajo son los sistemas educativos provinciales, a los que la institución formadora aporta innovación apoyo y, al tiempo que lo hace, se fortalece.

En segundo lugar, se destaca el carácter no homogeneizador, no compulsivo ni obligatorio con que el programa se puso en marcha y continúa su curso. Tratando de romper la lógica de formas de intervención política uniformadoras que omiten realidades y posibilidades locales, en este caso son las distintas provincias -a través de sus Direcciones de Educación Superior-interesadas en explorar esta nueva función

5 Desde entonces el programa se halla coordinado a nivel nacional por la profesora Beatriz Alen. En la actualidad, son los referentes provinciales de las Direcciones de Educación Superior quienes gestionan su desarrollo en las distintas jurisdicciones del país. 
del sistema formador, las responsables de seleccionar las instituciones formadoras en zonas diagnosticadas como prioritarias para su intervención, intentando cumplir con las necesidades cada vez más complejas y diversificadas que asume la formación continua de maestros y profesores. De otro modo, la generalización de las innovaciones muchas veces termina fagocitándolas (Viñao Frago, 2002).

En tercer lugar, se destaca el funcionamiento en red de la experiencia que comenzó "cara a cara” y que en la medida que el programa se fue extendiendo se concreta a través de la puesta en funcionamiento de un aula virtual en la que se dan a conocer las experiencias de las distintas provincias, se provee de recursos pedagógicos destinados a formadores y docentes, se organizan seminarios y foros coordinados por especialistas en los que se mantienen un fluido intercambio entre los formadores. Las instituciones formadoras cuentan, por su parte, con aulas virtuales para el acompañamiento. ${ }^{6}$

Finalmente, fue la documentación y sistematización de la experiencia transitada lo que permitió recuperarla, y ello representó no sólo una instancia reflexiva y productiva para quienes la protagonizaron y escrituraron, sino que además posibilitó la formulación de una política de desarrollo profesional docente nacional que capitalizó lo avanzado para hacerla potencialmente extensiva a todo el sistema formador del país. Quizás sea esta nota la que explica el afianzamiento y sostenimiento de las acciones emprendidas:

el logro de una mejora en el meollo mismo de la educación ha resultado ser el tipo más difícil de reforma, y en el futuro será resultado más de cambio internos creados por el conocimiento y la experiencia de los maestros que por las decisiones de políticos apartados de las aulas. (Tyack; Cuban, 2000, p. 262)

\section{LOS NUEVOS TIENEN LA PALABRA}

Más allá de las indagaciones permanentes que realizan las instituciones formadoras a partir del trabajo emprendido con los noveles docentes, se ha reconocido la importancia de recabar sistemáticamente las opiniones de los docentes que están involucrados en el Programa de Acompañamiento. Para estos fines, se ha diseñado y aplicado un cuestionario ${ }^{7}$ cuya finalidad fue recuperar la voz

6 Además de las instancias virtuales, el Ministerio de Educación Nacional, a través del INDF, apoya el trabajo de acompañamiento que realizan los formadores con actividades variadas (encuentros, seminarios, talleres, viajes al exterior) y provisión de recursos entre los que se encuentran publicaciones especializadas y material bibliográfico. Durante los años 2008-2011 el Área de Formación Docente de la OEI de Argentina ha cooperado en forma sostenida con este proyecto en sus aspectos pedagógicos y financieros, éste último gracias a dos subsidios obtenidos de la Agencia Española de Cooperación Internacional para el Desarrollo (AECID).

7 Con miras a obtener datos de este grupo específico se ha realizado una encuesta cuya aplicación fue de carácter individual y anónimo que, vía INFD, se acercó 
de los noveles sobre la tarea docente, la formación inicial y el propio programa. E1 propósito principal fue generar una instancia que recogiera sus voces, considerando las exigencias que la práctica docente les plantea. ¿En qué aspectos de su tarea los jóvenes maestros o profesores se sienten más seguros y en cuáles más débiles? ¿Cómo evalúan la formación recibida? ¿Qué valoran y qué consideran ineludible en una propuesta de acompañamiento? Como se ve, la indagación se interesó en conocer el punto de vista de los actores sobre la docencia, su formación y desarrollo profesional en contraste con su experiencia vivida en las escuelas como docentes.

\section{1) LO QUE SE SABE HACER Y LO QUE SE APRENDIÓ}

Interesa en este punto presentar los resultados obtenidos contemplando simultáneamente dos preguntas del cuestionario. La primera, se refiere a la percepción que tienen los noveles acerca de sus capacidades reales para enseñar. La segunda, remite a una evaluación de la formación recibida, en función de las exigencias que reconocen en la enseñanza. Dicho en otros términos, importa contemplar, al mismo tiempo, lo que los jóvenes maestros perciben que pueden hacer (o no) y lo que la formación docente les ha aportado (o no) para ello.

El 67\% de los docentes noveles percibe que está capacitado en los distintos contenidos de la enseñanza en la gran mayoría de las situaciones y el 32\% se reconoce de esta manera sólo en algunas situaciones. Los docentes sienten que saben los contenidos, siendo que en algunas provincias el porcentaje asciende por encima de la media. La formación inicial parece haberlos dejado bien provistos en este aspecto: el $44 \%$ considera que la calidad de la preparación profesional fue "muy buena" o "buena" (50\%), respecto al conocimiento de los temas a enseñar.

En relación a las estrategias de enseñanza, los datos manifiestan una tendencia inversa a la anterior. Sólo un 30\% de los docentes noveles considera que domina las estrategias para enseñar correctamente en la mayoría de las situaciones. La opinión sobre la calidad de la formación recibida en este ítem es consistente con la percepción que proviene del desempeño laboral: sólo un tercio de los docentes manifiesta que la formación fue muy buena en cuanto al manejo de las estrategias de enseñanza y un 50\% sólo buena.

Puntualmente sobre la planificación, el 68\% de los noveles percibe que está formado para anticipar lo que va a enseñar en la mayoría de las situaciones y el $28 \%$ sólo en algunas de ellas. El elevado reconocimiento que esta capacidad obtiene en el desempeño, disminuye al contemplar la influencia de la formación inicial: el $45 \%$ considera que la formación fue muy buena en este sentido y el $38 \%$ considera que fue buena.

a los institutos del profesorado de las distintas jurisdicciones que desarrollan el mencionado programa. Lo que aquí se presenta es una síntesis de las 177 encuestas que conforman la muestra, compuestas por docentes que inician su carrera profesional en las provincias de: Tucumán, Misiones, Córdoba, Neuquén, Salta, San Luis, Santa Fe y La Pampa. 
En comparación con las dimensiones hasta aquí presentadas, el manejo de la comunicación con los alumnos junto con la organización del grupo obtuvieron los porcentajes más elevados de respuesta. El 82\% y el 72\%, respectivamente, de los docentes noveles sienten que pueden manejar y organizar el grupo de alumnos en la gran mayoría de las situaciones de aula. Esta tendencia se refuerza al considerar que no suman un $2 \%$ la cantidad de docentes encuestados que manifestaron poder hacer estas tareas en pocas o ninguna situación de enseñanza. Los valores son altos en todas las provincias estudiadas; inclusive los más bajos, nunca son inferiores al $60 \%$ de los docentes.

Llama la atención en este caso que sólo el $40 \%$ de los noveles maestros reconoce las bondades de la formación en este aspecto. Es decir, si bien la gran mayoría percibe que puede manejar y organizar la clase, no tantos identifican esta capacidad con un aporte específico de la formación profesional. Quizás haya que tener en cuenta que el dominio de estos aspectos interactivos de la tarea docente frecuentemente se asocia con la experiencia y no tanto con la preparación formalizada. De todos modos, es un llamado de atención para formación inicial sobre todo cuando la intencionalidad declarada sea la de formar docentes con capacidades para la práctica.

Gráfico 1 - Capacidades percibidas en dimensiones de enseñanza

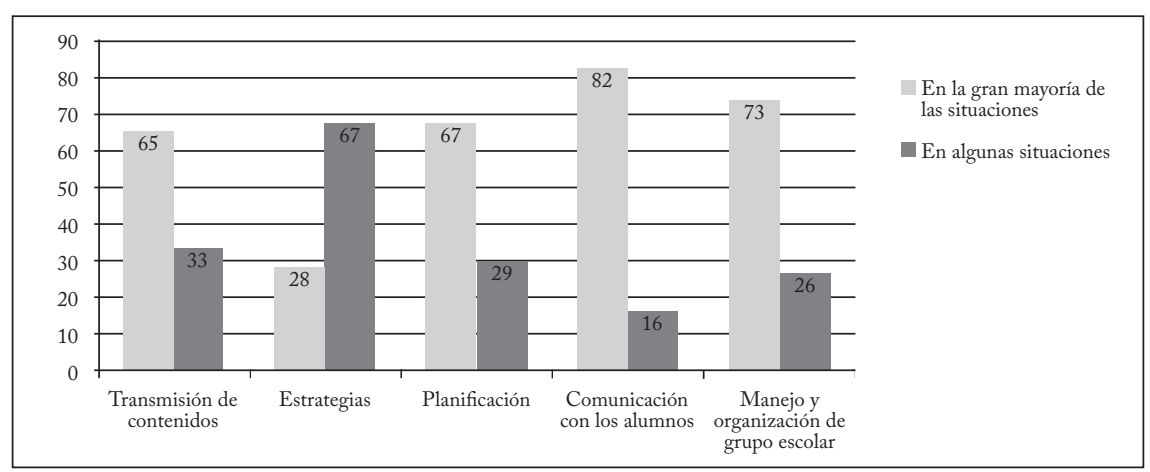

Fuente: Datos de la investigación académica.

Elaboración de la autora.

\section{2) PUNTOS FUERTES Y DEBILIDADES DE LOS MAESTROS, DE LA FORMACIÓN Y DEL ACOMPAÑAMIENTO}

Mientras el grupo funcione "normalmente" los jóvenes docentes parecen arreglárselas, a pesar de reconocer que la formación fue deficitaria en este aspecto interactivo o grupal. El panorama se complica cuando las situaciones de clase se muestran diversas y lo que se planifica o se espera parece no resultar. Más allá de casos puntuales, este tipo de situaciones pueden ser la norma en las escuelas del presente, sobre todo en las que comienzan a trabajar los maestros que se inician.

En las preguntas que indagaban acerca de sus "fortalezas y debilidades" como docentes, si bien la mayoría de los noveles (58\%) reconoce que lo mejor que pueden 
hacer como docente es enseñar/educar a sus alumnos, ${ }^{8}$ muchos de ellos se sienten débiles en la utilización de estrategias de enseñanza (31\%) y en el trabajo con niños que presentan dificultades o problemas especiales (23\%). El 17\% se siente flojo en áreas particulares de la enseñanza (desde materias curriculares y su enseñanza en los ciclos más altos hasta en contenidos de los espacios extra-curriculares) y un 10\% considera como falencia lograr el dominio del grupo.

\section{Gráfico 2 - Lo mejor que pueden hacer como docentes}

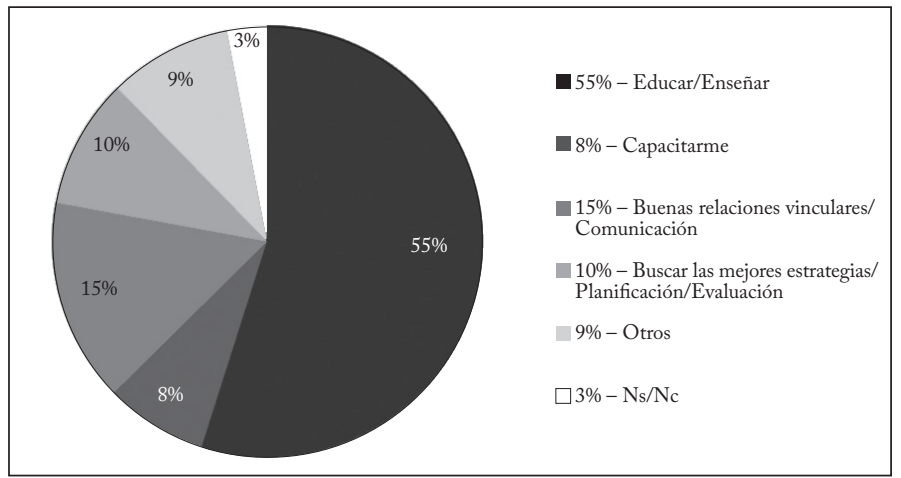

Fuente: Datos de la investigación académica. Elaboración de la autora.

\section{Gráfico 3 - Puntos más débiles a la hora de enseñar}

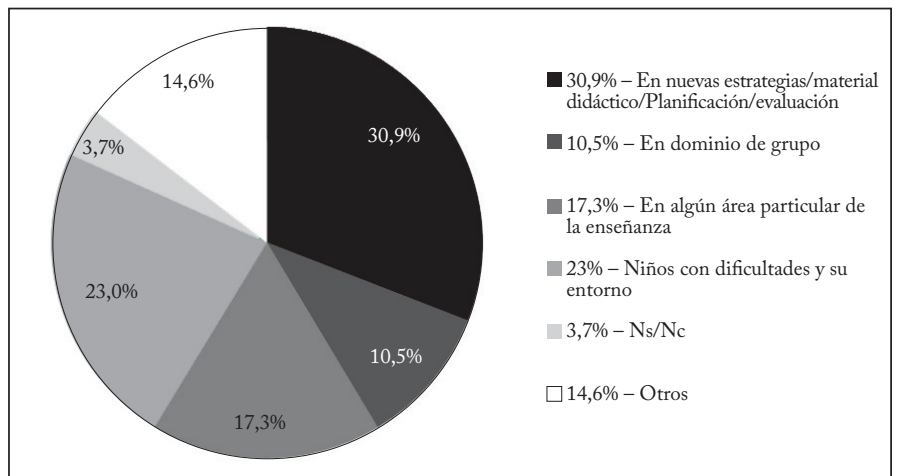

Fuente: Datos de la investigación académica.

Elaboración de la autora.

8 Los novatos consideran en su mayoría (31,1\%) que lo mejor que pueden hacer es enseñar, transmitir contenidos, saberes, etc. Un $12,8 \%$ identifica la transmisión con contenidos de orden moral y un $11,1 \%$ refiere su tarea a la educación y guía de los alumnos. Mientras este $55 \%$ remite a aspectos específicos de la educación y la enseñanza, el $15,3 \%$ de los docentes noveles considera que lo mejor que puede hacer es establecer buenas relaciones vinculares y manejar la comunicación con sus alumnos. 
En términos generales, los novatos valoran la formación recibida en los profesorados. Como vimos, los puntos fuertes (los más valorados por ellos desde su experiencia) son: el conocimiento de los temas a ser enseñados y la planificación, la preparación y el uso de material didáctico, la actualización de contenidos y bibliografía y la preparación para la práctica docente. Los novatos reconocen que la formación fue algo más débil en cuanto a haberles brindado estrategias didácticas y habilidades para atender las necesidades de los alumnos y particularmente para afrontar las de aquellos que presentan dificultades. La conducción del grupo de clase aparece, asimismo, entre las debilidades.

Los datos presentados van indicando que la formación docente inicial pierde fuerza o se desdibuja cuando aparecen los sujetos con quienes los docentes trabajan. Esta apreciación no es menor y lleva a pensar en una preparación formal que brinda conocimientos y habilidades que no pueden actualizarse en situaciones de clase reales.

Los novatos reconocen en su mayoría aspectos positivos del Programa de Acompañamiento vinculados con: la posibilidad de reflexionar, escuchar, compartir con colegas, obtener consejos, sentir seguridad, etc. Tres de cada diez, señalan que el asesoramiento y el acompañamiento a su tarea son aspectos que no deberían faltar en una propuesta formativa destinada a docentes que empiezan. Pero tampoco debería estar ausente, de acuerdo con sus apreciaciones, los aspectos referidos a: estrategias de enseñanza (23\%), cuestiones administrativas (19\%) y tratamiento de casos difíciles y contextos particulares (17\%). Tanto el tratamiento de casos difíciles, como la resolución de problemas de conducta, el manejo del grupo y la organización de la actividad docente, parecen ser los temas de más urgentes que aún quedan pendientes.

\section{Gráfico 4-Ayuda que proporcionó el Programa de Acompañamiento}

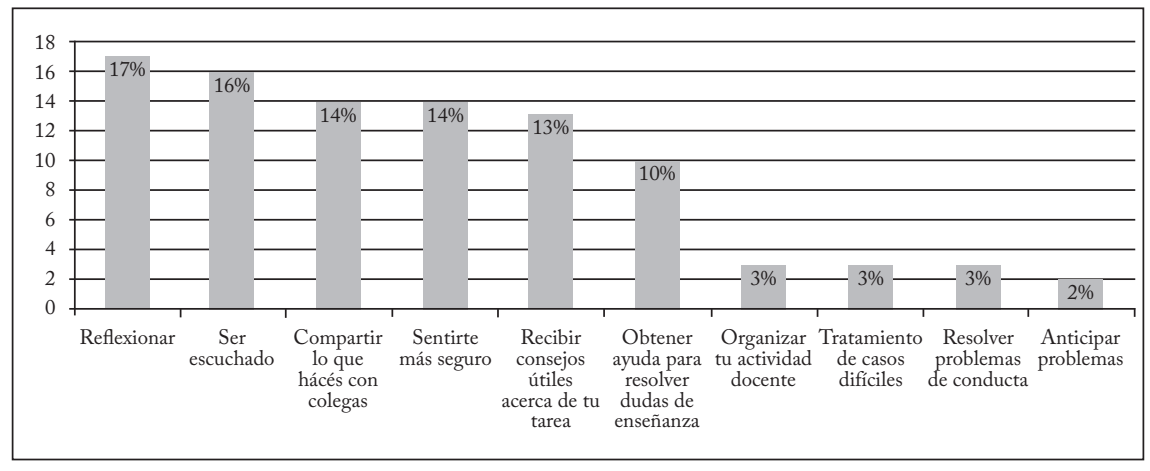

Fuente: Datos de la investigación académica.

Elaboración de la autora. 


\section{Gráfico 5 - Aspectos que no deben faltar en una propuesta formativa destinada a novatos}

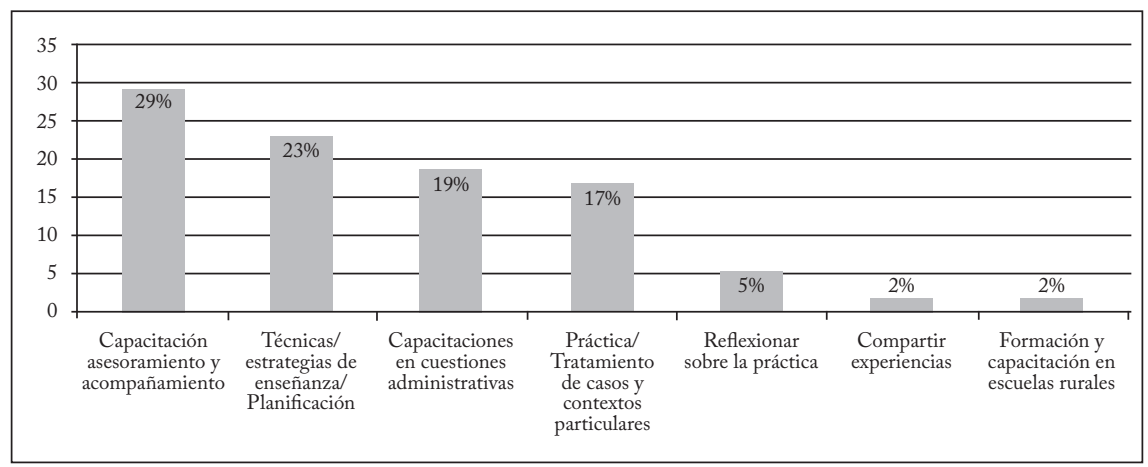

Fuente: Datos de la investigación académica.

Elaboración de la autora.

Más allá de que las preguntas del cuestionario remitían a las instancias formales de formación y desarrollo profesional docente, en distintas respuestas aparece como tendencia la salida individual como una forma de afrontar los problemas que los noveles detectan en su quehacer. Un número importante (21\%) asocia el ser buen maestro con el compromiso de cada uno. Asimismo, un porcentaje de ellos (10\%) considera que lo mejor que puede hacer es buscar por su cuenta las mejores estrategias y atender la planificación y la evaluación. Para un $8 \%$ lo mejor es capacitarse.

\section{CONCLUSIÓN: FORTALECER EL OFICIO}

Hemos sostenido, al principio del texto, que todo comienzo laboral es difícil. Que la dificultad parece acrecentarse en aquellas actividades en que están comprometidas las personas. Que la fragilidad institucional propia de estos tiempos, quita respaldo a la tarea y sobre-expone a quienes tienen que efectivizarla. En el caso de la docencia, mencionamos condiciones laborales propias de los inicios que suman a la lista de problemas presentados.

Ante este panorama, podemos añorar un pasado que no es (más bien ya fue), aumentar los niveles personales de "resistencia" o "exigencia" (haciendo o esperando que los maestros sean fuertes, resistentes, conquistadores, estrategas o carismáticos) o simplemente "fortalecer el oficio". Tal es uno de los cursos de acción posibles para habitar las instituciones en esta etapa de la modernidad. Ello significa "que el trabajo sea objetivable y que el profesional pueda decir: ésta es mi obra, éste es el resultado de mi actividad, puedo mostrarlo y demostrarlo" (Dubet, 2006, p. 443). El oficio es concebido como "la capacidad de producir algo, de conocerlo y de hacerlo conocer" (idem, p. 444).Para los casos en que se oficia con personas, puede resultar más complejo identificar el "producto" de la propia intervención o actividad. ¿Cómo sabemos si lo 
que el otro sabe, hace, aprendió, es producto de un obrar/ accionar específico; si fue producido por mí, por "mi”" enseñanza? Es más difícil pero no es imposible identificar el propio trabajo o el del equipo al que se pertenece. Basta el indicio, la prueba que no siempre se asocia con un resultado mensurable. Pero el reconocimiento no es suficiente. Hoy, más que nunca, es necesario mostrar ese producto (esa obra) públicamente.

Apoyarse en el oficio, percibir que se tiene oficio, tranquiliza y legitima lo que se hace. De otro modo, cuando el quehacer se vuelve un "ideal", algo muy frecuente entre los docentes (y aún más en los novatos), se vuelve impracticable, sobre todo en las condiciones actuales. Así, el sentimiento omnipotente de poderlo todo se torna, ante la dificultad, en la impotencia de no poder hacer nada (Alliaud; Antelo, 2009). Movidos por un ideal, o una alta expectativa inicial, el "choque" con la realidad frecuentemente lleva a los nuevos a experimentar sentimientos de fracaso:

Con 20 años de edad, mi título en mano, un buen promedio y llena de expectativas, me dirigía hacia mi nueva escuela. [...] Muchos de mis alumnos provenían de hogares humildes, familias destruidas, condiciones de vida precarias, algunos pasaban más tiempo en la calle que en sus casas. [...] Algunos eran más grandes que yo en estatura y edad, parecía haberme bloqueado, miraba atónita cómo se desbarataban mis estrategias, me sorprendían, me superaba, me sentía un fracaso. (Testimonio de una maestra novel)

Los resultados de distintas investigaciones (Alliaud, 2004a, 2004b) han demostrado que los maestros novatos parecen asumir responsablemente el mandato original del proyecto de educar a las nuevas generaciones (en un sentido redentor y salvador), lo cual los engrandece y les da felicidad, pero los deja bastante indefensos a la hora de accionar. Esta marca de origen que funciona como un ideal, convive con la sensación de no poder enseñar.

Las voces de los maestros novatos presentados en esta oportunidad, dan cuenta de la debilidad que ellos perciben para poder hacer, para poder obrar con otros y sobre otros, en tanto reconocen la falta de estrategias pedagógicas, sobre todo cuando tienen que enseñar a alumnos que presentan problemáticas particulares, distintos al "tipo" esperado. Así, los jóvenes perciben que saben, pero esos saberes no parecen ser suficientes para operar, intervenir, accionar, enseñar con personas y en situaciones concretas.

Si pretendemos que los docentes puedan enseñar, tendríamos que asegurar la posesión de esa capacidad que les permitiría operar, intervenir, accionar, sin sentir la amenaza de desaparecer o quedar paralizados (sin saber qué hacer) en las situaciones cotidianas. Estas apreciaciones nos conducen directamente a la formación, tanto inicial como continua o permanente.

¿Cómo transmitir el oficio de enseñar? ¿Cómo lograr ese sentimiento de propiedad del oficio, esa capacidad productiva en los maestros? ¿Cómo asegurarlo en los noveles que todavía no cuentan con un repertorio construido a partir de la propia experiencia? Son preguntas imprescindibles que habría que contemplar a la hora de tomar decisiones en las instancias de formación y desarrollo profesional 
docente. Atendiendo particularmente a esta última y al trabajo con maestros noveles, tendríamos que considerar ciertos rasgos del proceso formativo que apuntaran hacia formas de fortalecimiento del oficio, en el sentido que lo hemos definido anteriormente y que el programa de Argentina en su mayoría contempla.

En primer lugar, el desarrollo profesional con noveles supone que el trabajo formativo no se desarrolla con alumnos, sino con colegas. Son colegas "con oficio" los que acompañan, guían, ayudan, a quienes se están iniciando. Los que acompañan la enseñanza, dialogan con un nuevo colega, y ese intercambio contempla no sólo los aspectos técnicos de la tarea sino, también, las competencias sociales y capacidades de "adaptación" a los escenarios laborales diversos y cambiantes.

En segundo lugar, el oficio (la enseñanza en nuestro caso) es lo que nuclea las actividades que se desarrollan. Las problemáticas particulares que la tarea docente plantea (les plantea a los nuevos) son tomadas como punto de partida, pero son definidas o re-definidas en función de este núcleo principal que define a la docencia y su identidad. Así entendida, la enseñanza no es sólo el objetivo o la meta a alcanzar, sino que constituye el método del acompañamiento y se utiliza como herramienta a lo largo de todo el proceso.

En tercer lugar, la puesta a disposición de recursos o herramientas que ayuden a los noveles en su proceso de producción, se entiende no como una capacitación en torno a habilidades específicas solamente, sino como una formación que está al servicio de la persona, de su posibilidad y potencialidad y que, por lo tanto, la libera, la emancipa para poder obrar en un sentido autónomo y creativo en cualquier circunstancia.

En cuarto lugar, el modelo de acompañamiento supone el tratamiento de cuestiones teóricas, pero éstas cobran sentido cuando son necesarias para afrontar y resolver problemas que se plantean en situaciones prácticas. En este caso, el distanciamiento de la escena escolar, permite que se puedan imaginar cursos de acción alternativos, líneas de intervención y, de este modo, aprender de aquello que en primera instancia se presenta como difícil o irresoluble. Muchas veces ante los casos más difíciles, se inventan los métodos más originales que luego pueden ser retomados y probados por todos. ${ }^{9}$

En quinto lugar, tanto en las relaciones que se establecen entre los formadores y los noveles como las que se generan entre ellos, predomina la colaboración. Para Sennett (2009) y en contra de lo que suele creerse en el mundo moderno, la colaboración antes que la competencia es lo que favorece el aumento de la productividad. Cuando desaparece la cooperación y predominan los solistas, el trabajo se degrada. Uno se forma al formar parte de la vida de un grupo de trabajo, sostiene Dubet (2006). La relación colaborativa se focaliza en la tarea más que en la persona o, mejor, se centra en las personas en tanto sujetos que están compartiendo un proceso de formación/ producción y que para hacerlo se requieren mutuamente.

9 En su libro El artesano, Richard Sennett (2009) plantea que antes que luchar contra esas experiencias, todo artesano tendría que poder aprender de ellas. 
Son los mencionados sólo algunos de los rasgos que, a nuestro juicio, requieren ser contemplados en una propuesta de formación y desarrollo profesional para docentes noveles. Abrir la discusión en torno a ellos, enriquecerlos, problematizarlos a partir de las experiencias llevadas a cabo en distintos países de la región, puede contribuir a afianzar prácticas pedagógicas y decisiones políticas que las nuevas condiciones de ejercicio profesional de la docencia requieren. Espacios, como el que tenemos la oportunidad de participar esta vez, favorecen el encuentro entre especialistas/protagonistas de experiencias y abren una fecunda posibilidad para avanzar en el sentido aludido.

\section{REFERENCIAS}

Alen, Beatriz; Allegroni, Andrés. Acompañar los primeros pasos en la docencia, explorar una nueva práctica de formación. Tomo 1. Buenos Aires: Ministerio de Educación de la Nación; Instituto Nacional de Formación Docente (INFD)/OEI/AECID, 2009.

Alliaud, Andrea. La biografía escolar en el desempeño profesional de los docentes noveles. 2004. 292f. Tesis (Doctorado en Educación) - Facultad de Filosofía y Letras, Universidad de Buenos Aires, Buenos Aires, 2004a.

. La escuela y los docentes: ¿Eterno retorno o permanencia constante? Apuntes para abordar una particular relación desde una perspectiva biográfica. Cuaderno de Pedagogía, Rosario: Centro de Estudios en Pedagogía Crítica, El Zorzal, año 7, n. 12, p. 91-106, 2004b.

; Antelo, Estanislao. Los gajes del oficio. Enseñanza. Pedagogía y Formación. Buenos Aires: Aique, 2009.

Dubet, François. El declive de la institución. Profesiones, sujetos e individuos en la modernidad. Barcelona: Gedisa, 2006.

Sennett, Richard. El artesano. Barcelona: Anagrama, 2009.

Track, David; Cuban, Larry. En búsqueda de la utopía. Un siglo de reformas de las escuelas públicas. México: Fondo de Cultura Económica, 2000.

VIñao Frago, Antonio. Sistemas educativos, culturas escolares y reformas. Continuidades y cambios. Madrid: Morata, 2002.

\section{SOBRE LA AUTORA}

Andrea Alliaud es doctora en educación por la Universidad de Buenos Aires (UBA). Profesora de la misma institución.

E-mail: andrealliaud@ciudad.com.ar

Recebido em agosto de 2011

Aprovado em março de 2013 


\section{ANDREA ALLIAUD}

\section{Las políticas de desarrollo profesional del profesor principiante en el Programa de Acompañamiento de Docentes Noveles en su Primera Inserción Laboral de Argentina}

Considerando las dificultades que tienen que enfrentar los docentes cuando se inician en su profesión, particularmente en la actualidad, este trabajo presenta ciertas alternativas de intervención política y pedagógica tomando como referencia el Programa de Acompañamiento de Docentes Noveles en su Primera Inserción Laboral de Argentina. En una primera parte, se exponen los rasgos fundamentales de este programa que constituye una de las líneas de desarrollo profesional docente a nivel nacional. La lógica del acompañar y los distintos dispositivos implementados, las experiencias locales como insumos para la definición de políticas públicas, son algunos de ellos. A continuación, se presentan y analizan las opiniones de los docentes noveles que han recibido acompañamiento en su primera inserción laboral sobre las fortalezas y debilidades que ellos perciben a la hora de enseñar, la valoración de la formación recibida y la propuesta de acompañamiento en sí misma. Finalmente, se ponen en consideración una serie de características que se tendrían que contemplar por cualquier propuesta formativa que apunte al fortalecimiento del oficio de enseñar.

Palabras clave: desarrollo profesional; docentes noveles; formación docente. 


\section{Policies of professional development for beginning teachers in the Programme for Supervision of Inexperienced Teachers Entering the Labor Market in Argentina}

Considering the difficulties faced by teachers when they begin working, particularly at present times, this article presents some alternatives for political and pedagogical interventions taking as a reference the Programme for Supervision of Inexperienced Teachers Entering the Labor Market in Argentina. In the first part, the main features of this programme are exposed. It is one of the national lines of teacher professional development. Supervision, the different devices implemented and local experiences as inputs for defining public policies are some of those lines. Furthermore, opinions of inexperienced teachers who have been followed in their entry in the labor market are presented and analysed, with emphasis on strengths and weaknesses perceived when teaching, the value of the training received and the supervision proposal itself. In the end, several characteristics which should be considered by every training proposal aimed at strengthening the teaching job are examined.

Keywords: professional development; beginning teachers; teacher training.

\section{As políticas de desenvolvimento profissional do professor principiante no Programa de Acompanhamento de Docentes Inexperientes na sua Primeira Inserção Laboral da Argentina}

Levando em consideração as dificuldades que os docentes têm de enfrentar quando iniciam a sua profissão, principalmente nos dias de hoje, este trabalho apresenta certas alternativas de intervenção politica e pedagógica, tomando como referência o Programa de Acompanhamento de Docentes Inexperientes na sua Primeira Inserção Laboral da Argentina. Na primeira parte, são exploradas as características fundamentais desse programa, que constitui uma das linhas de desenvolvimento profissional docente em nivel nacional. A lógica de acompanhar e os diferentes dispositivos implementados e as experiências locais como insumos para a definição de politicas públicas são alguns exemplos. $\mathrm{Na}$ continuação, apresentam-se e são analizadas as opinióes dos docentes inexperientes que têm recebido acompanhamento na sua primeira inserção laboral, com destaque para os pontos fortes e as debilidades que eles percebem no momento de ensinar, a valorização da formação recebida e a própria proposta de acompanhamento. Finalmente, coloca-se em consideração uma série de caracteristicas que teria de ser contemplada por qualquer proposta formativa que aponte o fortalecimento do ofício de ensinar.

Palavras-chave: desenvolvimento profissional; docentes inexperientes; formação docente. 